\title{
Twin-assisted growth mechanism of heterogeneous interfaces between gold nanoparticles and nominally stable substrates
}

\author{
Fang Liu ${ }^{1}$, Dong Yue Xie ${ }^{1}$, Yong-sheng Fu${ }^{1}$, Guo-zhen Zhu ${ }^{1}$ \\ 1. State Key Laboratory of Metal Matrix Composites, School of Materials Science and Engineering, \\ Shanghai Jiao Tong University, Shanghai, 200240, P. R. China.
}

Due to the attractive applications of gold nanoparticles in fields of biology and optics [1], gold nanoparticles supported on the oriented single-crystal substrate including sapphire and silicon have been extensively investigated for decades [2-5]. However, the heterogeneous interfacial structures between gold nanoparticles and substrates and the consequent properties have not been clarified yet. In this paper, we reported the twin-assisted growth mechanism, corresponding to the abnormal regrowth of nominally stable substrates underneath dewetted Au nanoparticles. Such regrowth phenomenon was reported recently and linked for the formation of special interfacial monolayers [5-6]. We believed that the clarification of the interfacial structures and the underlying regrowth mechanism can promote the synthesis of gold-oxide nanostructure and provide a new view for the migration of heterogeneous interface and the structural evolution of interfacial complexions.

Thin gold films were sputtered on the (111) oriented spinel single-crystal (MTI corp.). The samples, which were sealed in glass tubes filled with $99.999 \%$ purity argon gas, were annealed to the temperature range of $900-1110{ }^{\circ} \mathrm{C}$ for $10-90 \mathrm{~min}$, and then cooled to room temperature within $5 \mathrm{~h}$. The morphology of gold nanoparticles and intricate nanostructures were examined by scanning electron microscopy (SEM). The crystallographic orientations between gold nanoparticles and the substrate were investigated through a Bruker X-ray Instrument. The atomic interfacial structures were characterized using scanning transmission electron microscopy (STEM) at $200 \mathrm{kV}$ (JEOL-ARM200F). The cross-sectional samples were prepared by the Focused Ion Beam (FIB) technique (FEI Versa 3D).

Figure 1 shows the abnormal regrowth of nominally stable substrate, which was related to the in-plane texture of dewetted gold nanoparticles. The $\{111\}$ Au pole figure detected from the gold nanoparticles indicates four preferential orientation relationships (OR) between gold nanoparticles and the substrate, supported by the comparison between the experimental and simulated pole figures. The above results invoke a question as to why irregular crystallographic planes, e.g. $\{11111\}$, are selected parallel to the $\{111\}$ spinel surface, since $\{111\}$ Au surface has a good match with the spinel substrate. The preferential ORs, namely ORa-ORd, are the first-order and second-order twined orientations with each other (as listed in table 1). Thus, the coexistence of two or even more orientations within one single nanoparticles through twinning can be our answer. Herein, the morphology of gold nanoparticles is modified by twinning, which can decrease the surface energy through introducing more low-energy facets. The unique interfacial monolayers, including re-arranged gold monolayers as shown in STEM-HAADF (Zcontrast) images in Figure 2 (b)-(c), were reconstructed at the interface. In corresponding to the twinned structure, defects, such as the various steps (figure 2 (d)-(e)), present at the interfacial monolayers, which can be the underlying reason for the migration of the interfaces and regrowth of the substrates.

The detailed atomic arrangements of $\mathrm{Au}-\mathrm{MgAl}_{2} \mathrm{O}_{4}$ interfacial monolayers, in addition to their evolution with temperature, are currently being studied by the STEM technique and the density functional theory (DFT) computation. 


\section{References:}

[1] J. Pérez-Juste, et al, Coordination Chemistry Reviews 249(2005), p. 1870-1901.

[2] R. Wagner, W. Ellis, Applied Physics Letters 4(1964), p. 89-90.

[3] M. Baram, D. Chatain, W. D. Kaplan, Science 332(2011), p. 206-209.

[4] H. Sadan, W. D. Kaplan, Journal of Materials Science 41(2006), p. 5099-5107.

[5] G.-z. Zhu, et al, Applied Physics Letters 105(2014), p. 231607.

[6] F. Liu, et al, Materials Characterization 113(2016), p. 67-70.

[7] We thank Ms. Tahereh Majdi for beneficial discussions and texture measurement. The financial support from the National Science Foundation of China (No. 51401124) is greatly acknowledged.
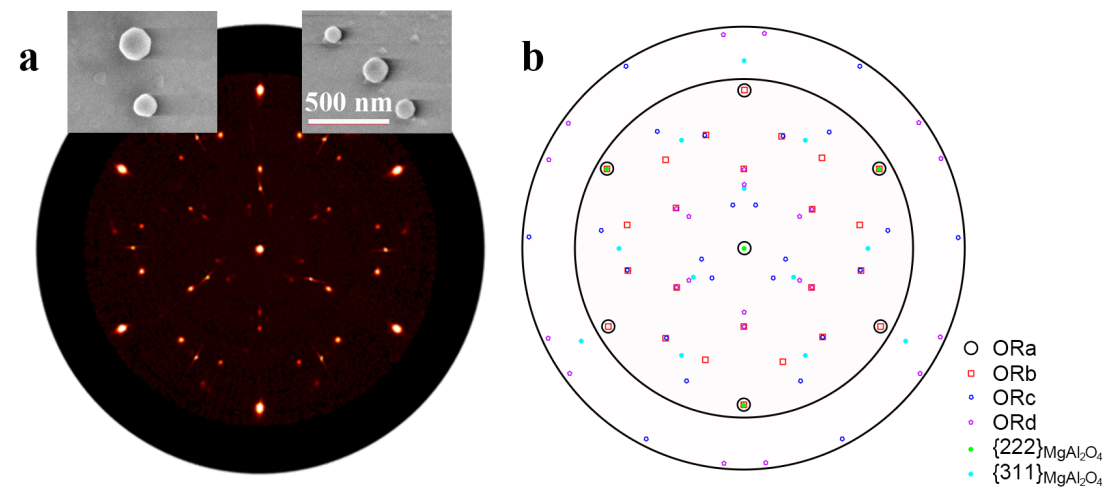

Figure 1. (a) The $\{111\}$ Au pole figure measured by X-ray diffraction techniques. The inserted SEM images show the morphology of gold nanoparticles, the top view on left and the side view with $40^{\circ}$ tilt angle on right. (b) the simulated pole figure using MATLAB codes based on the listed ORs in Table 1.

Table 1 The preferential orientation relationships between gold nanoparticles and the $\{111\}$ spinel surface

\begin{tabular}{|c|c|}
\hline ORa: $\{111\}_{\mathrm{Au}} / /\{111\}_{\mathrm{MgAl}_{2} \mathrm{O}_{4}} \&<1 \overline{1} 0>_{\mathrm{Au}} / /<1 \overline{1} 0>_{\mathrm{MgAl}_{2} \mathrm{O}_{4}}$ & ORb: $\{115\}_{\mathrm{Au}} / /\{11\}_{\mathrm{MgAl}_{2} \mathrm{O}_{4}} \&<1 \overline{1} 0>_{\mathrm{Au}} / /<1 \overline{1} 0>_{\mathrm{MgAl}_{2} \mathrm{O}_{4}}$ \\
\hline ORc: $\left\{\begin{array}{llll}5 & 7 & 13\end{array}\right\}_{\mathrm{Au}} / /\{111\}_{\mathrm{MgAl}_{2} \mathrm{O}_{4}} \&<\overline{4} 11>_{\mathrm{Au}} / /<1 \overline{1} 0>_{\mathrm{MgAl}_{2} \mathrm{O}_{4}}$ & ORd: $\{11111\}_{\mathrm{Au}^{\prime}} / /\{11\}_{\mathrm{MgAl}_{2} \mathrm{O}_{4}} \&<1 \overline{1} 0>_{\mathrm{Au}} / /<1 \overline{1} 0>_{\mathrm{MgAl}_{2} \mathrm{O}_{4}}$ \\
\hline
\end{tabular}
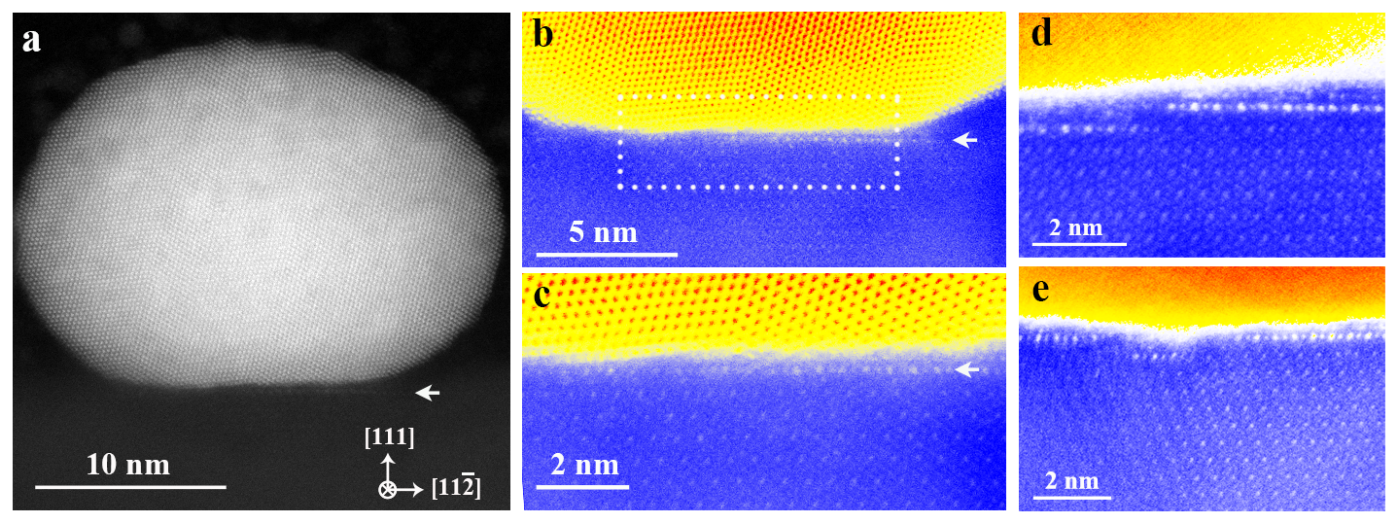

Figure 2. STEM-HAADF images showing the atomic structure of $\mathrm{Au}-\mathrm{MgAl}_{2} \mathrm{O}_{4}$ interfaces. (a) the typical gold nanoparticle. (c) an enlarged view of the boxed region in (b), showing the nucleation of the interfacial monolayers at defects created by twinning. (d)-(e) various steps on the interface. 